\title{
Authors' Reply to Gandjour: “Are Current Cost-Effectiveness Thresholds for Low- and Middle-Income Countries Useful? Examples from the World of Vaccines"
}

\author{
A. T. Newall $\cdot$ M. Jit $\cdot$ R. Hutubessy
}

Published online: 6 November 2014

(C) Springer International Publishing Switzerland 2014

We thank Gandjour [1] for his interest in our article [2] and for highlighting the 'proportional rule' [3] approach as another alternative to setting cost-effectiveness thresholds. However, we feel that its use for low- and middle-income countries (LMICs) raises some important questions.

The approach as described by Gandjour [1] essentially proposes a separate decision threshold for each therapeutic area, with the threshold being dependent on the incremental cost effectiveness of the existing intervention/s within that area. Whereby, new interventions within the therapeutic area would not be deemed 'cost effective' unless they provided incremental health gains at a lower incremental cost than previously funded interventions.

This approach may help to ensure that investment decisions within a given therapeutic area will always be more efficient than those previously taken (i.e. that new interventions will provide more health gains for each additional dollar spent). However, particularly in LMICs, it is unlikely that all existing interventions within different therapeutic areas can be assumed to offer value for money

A. T. Newall $(\square)$

School of Public Health and Community Medicine, Faculty of Medicine, UNSW Australia, Sydney, NSW 2052, Australia

e-mail: a.newall@unsw.edu.au

M. Jit

Department of Infectious Disease Epidemiology, London School of Hygiene and Tropical Medicine, London, UK

M. Jit

Modelling and Economics Unit, Public Health England,

London, UK

R. Hutubessy

Initiative for Vaccine Research, World Health Organization,

Geneva, Switzerland or that the allocation of resources to the existing mix of interventions between therapeutic areas will be efficient [4]. As a consequence, there may be a danger of perpetuating inefficient resource allocation by applying proportional rule thresholds derived from past funding decisions.

The estimation of cost-effectiveness thresholds for LMICs is a complex area with no easy answers. However, as stated in our article, a major aim of thresholds in LMICs should be to ensure that scarce resources are not allocated to interventions that offer poor value compared with other potential health spending choices. It is not clear to us that the proportional rule would achieve this in LMICs.

Acknowledgments $\mathrm{RH}$ is a staff member of the World Health Organization. The views expressed are that of the author and do not necessarily represent the views of the World Health Organization.

Conflict of interest ATN has, in the past, received research funding from a vaccine manufacturer for other previous projects.

\section{References}

1. Gandjour A. Comment on: "Are current cost-effectiveness thresholds for Low- and middle-income countries useful? Examples from the world of vaccines". Pharmacoeconomics. 2014. doi:10.1007/s40273-014-0221-3.

2. Newall AT, Jit M, Hutubessy R. Are current cost-effectiveness thresholds for low- and middle-income countries useful? Examples from the world of vaccines. Pharmacoeconomics. 2014;32(6): 525-31.

3. Gandjour A. Germany's decision rule for setting ceiling prices of drugs: a comparative analysis with other decision rules. Appl Health Econ Health Policy. 2011;9(2):65-71.

4. Baltussen R. Priority setting of public spending in developing countries: do not try to do everything for everybody. Health Policy. 2006;78(2-3):149-56. 